\title{
DeepSIL: A Software-in-the-Loop Framework for Evaluating Motion Planning Schemes Using Multiple Trajectory Prediction Networks*
}

\author{
Jan Strohbeck ${ }^{1, \dagger}$, Johannes Müller ${ }^{1, \dagger}$, Adrian Holzbock ${ }^{1}$, and Michael Buchholz ${ }^{1}$
}

\begin{abstract}
Testing and verification is still an open issue on the way to fully automated driving. Simulations can help to reduce the required testing efforts, however, classical simulators based on physical models and heuristics, such as the intelligent driver model (IDM), show limited model accuracy on a microscopic scenario level. In turn, learning-based driver models are often capable to predict human driver's behavior accurately, but are difficult to tailor such that they follow an intended scenario description. In this work, we propose a software-in-the-loop framework to combine a learned model with a rule-based logic layer and a kinematic vehicle model of a classical traffic simulator. Thus, the merits of both, classical simulators and learning-based models are exploited. We demonstrate with a case study of evaluating a motion planning scheme that the simulator fits well with the needs of testing such methods. Furthermore, we show by experiments with real-world traffic data from a traffic surveillance system that the proposed simulator yields realistic behavior of the simulated road users.
\end{abstract}

\section{INTRODUCTION}

With increasing level of automation, the responsibility is more and more transferred from the driver to the automated vehicle. Thus, safety assessment becomes particularly important. However, due to the broad variety of possible traffic situations and the tough safety requirements an automated vehicle has to meet, an infeasibly high number of test kilometers would be required to statistically verify the safety of automated vehicles [1]. Scenario-based testing in combination with simulation-based verification techniques can significantly reduce the required testing efforts. However, this requires reliable and highly realistic simulations of a broad variety of traffic scenarios. Using datasets provides realistic input data, but cannot be used for closed-loop tasks like motion planning, since the input data needs to be adapted depending on the decisions of previous steps. In turn, classical simulators like SUMO [2] use physical models like a kinematic bicycle model in combination with heuristic driver models like the intelligent driver model (IDM) [3] to simulate the motion of traffic participants. Yet, while these models are accurate for many situations, they tend to fail in

\footnotetext{
*Part of this work was financially supported by the Federal Ministry of Economic Affairs and Energy of Germany within the program "New Vehicle and System Technologies" (project LUKAS, grant number 19A20004F). Part of this work has been conducted as part of ICT4CART project which has received funding from the European Union's Horizon 2020 research $\&$ innovation program under grant agreement No. 768953. Content reflects only the authors' view and European Commission is not responsible for any use that may be made of the information it contains.

$\dagger$ J. Strohbeck and J. Müller are both first authors with equal contribution.

1 All authors are with the Institute of Measurement, Control and Microtechnology, Ulm University, D-89081 Ulm, Germany \{jan.strohbeck, johannes-christian.mueller, adrian.holzbock, michael.buchholz\}@uni-ulm.de
}

realistically modeling the interaction between multiple traffic participants. Recently, deep learning methods like generative adversarial imitation learning (GAIL) [4] and variants thereof [5], [6], have been proposed to describe the behavior of other road participants more realistically. However, while we acknowledge the recent achievements, these approaches are still in their infancies and often suffer from poor generalization to multi-agent scenarios and performance issues [6]. Furthermore, these approaches have mostly been applied to the NGSIM dataset [7] only, which covers a limited spectrum of possible traffic scenarios. Moreover, as stated in [5], it is still up to future work to integrate these learned models into a simulator for safety verification.

In this work, we propose DeepSIL, a software-in-the-loop (SIL) framework that combines a deep neural network for multiple trajectory prediction (MTP), e.g. [8], with a classical rule-based logic layer and a kinematic bicycle model. By using a kinematic model of the vehicles, a physically realistic motion of the simulated vehicles is ensured. Furthermore, using a rule-based logic layer with a Monte-Carlo randomized sampling makes the DeepSIL simulator closely follow the scenario description of the simulation and supports an efficient evaluation. At the same time, by using the deep MTP network, the behavior of the road users is learned from real-world examples and, thus, tailors the behavior of the simulated vehicles as realistic as possible. Thus, the merits of both, learning based methods and classical simulators, are combined.

To demonstrate the usefulness of the proposed DeepSIL framework, a case study is conducted that exemplarily shows how the framework can be used to evaluate a motion planning scheme. Furthermore, by comparing recorded realworld scenarios with traffic scenarios generated by the simulator, it is shown that DeepSIL yields realistic trajectories of the simulated vehicles. Besides, since the MTP network is trained on the Argoverse dataset [9], which was recorded in large cities in the USA, while the evaluation is shown on data recorded at a suburban T-junction in Germany, the generalization capability of our approach is demonstrated.

\section{A. Contributions}

Our contributions can be summarized as follows:

- To the best of our knowledge, we are the first to use a deep MTP network for SIL testing.

- We propose a deep SIL architecture that combines the merits of both, classical simulators and deep-learning methods. 
- Compared to classical simulators, the simulated behavior is more realistic, while the proposed simulator is still capable to closely follow the scenario description.

- The usefulness of the approach is demonstrated with the case study of evaluating a motion planning scheme.

\section{B. Related Work}

Simulators for automated driving have been studied intensively in the recent years. From a safety and verification perspective, it has been studied how to select scenarios, e.g. [10], and how simulative scenario-based verification methods can help to approve the safety of automated driving. An overview on scenario-based verification can be found in [1]. In this context, several simulators, e.g. [11], [12], have been proposed, mostly based on heuristics and rough physical models. One particular famous combination of heuristics and rough physical modeling is the intelligent driver model (IDM) [3], which has been used in several variations for many simulators. For traffic management and similar highlevel tasks, large scale simulators like SUMO [2] have been proposed, which also rely on the IDM or similar models due to their low computational burden. However, the IDM can only roughly describe the interaction between road users and, thus, the resulting trajectories are not very realistic.

Other simulators also simulate sensor data for e.g. cameras and LiDAR, like CARLA [13], or concentrate on simulating cooperative motion planning algorithms, like CoInCarSim [14]. However, all of these simulators do not focus on generating realistic behavior for road users that are not controlled by motion planning algorithms, and instead rely on simple models.

Using scenario databases like from [15] or datasets, like [9] can help to identify critical or challenging situations and are useful for the anticipation of other road users' behavior. However, recorded data cannot be applied to closed-loop tasks, since the recorded objects do not interact with the vehicles controlled by the planning algorithm.

In turn, datasets can be used for the training of neural networks, which show the ability to interact. For the prediction of other road users, recently, several deep-learning methods have been proposed, e.g. [8], [16]. Nevertheless, while these neural networks do produce highly realistic trajectories, they do not offer the possibility to manipulate the trajectories, i.e. the predicted trajectories do not necessarily comply with a prescribed scenario. This, however, is required for scenariobased verification methods. On the other hand, inverse reinforcement learning and imitation learning methods, such as GAIL [4] and variations thereof [5], [6], have been designed to actually control an automated vehicle and, thus, are capable of both, to comply with a scenario script book and to realistically mimic other road users. However, so far, these methods have mostly been tested on the NGSIM dataset [17], which comes with a limited variation of scenarios. Furthermore, several shortcomings, like performance issues or difficulties to work for multiple agents in parallel have been reported [6].

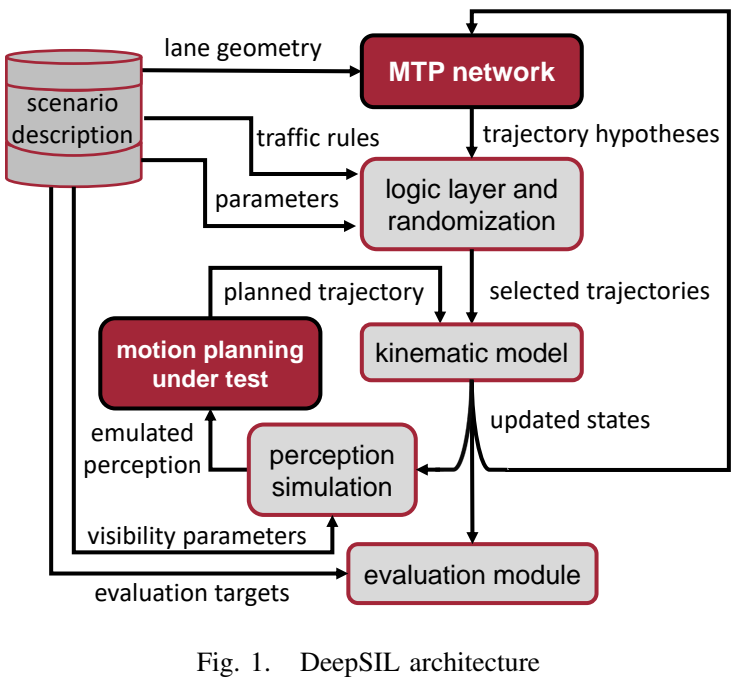

\section{SIMULATION FRAMEWORK}

The main goal of DeepSIL is to provide a realistically simulated environment to evaluate a motion planning software under test (MPUT). To realize this, we choose a hybrid approach: on the one hand, DeepSIL consists of a deep MTP network to realistically simulate the motion behavior of the road users in the scenario. On the other hand, DeepSIL comprises the non-learning-based logic layer, kinematic models and a Kalman filter to tailor the simulated driving behavior according to the scenario description. Furthermore, the nonlearning-based evaluation module measures the performance of the MPUT. The DeepSIL architecture is sketched in Fig. 1, and the simulation flow is shown in Algorithm 1.

The simulation is initialized with a scenario description $\mathcal{D}$ which is loaded from a file. Then, as the first step of the simulation iteration, the perception simulation generates an environment model from the states of the simulated agents $\mathcal{S}_{\boldsymbol{x}}$ and sends it to the MPUT. The MPUT uses this as input to plan its next trajectory. Next, the MTP network predicts multiple possible trajectories $\hat{\mathcal{T}}$ and their likelihood $p(\hat{\mathcal{T}})$ for the remaining simulated agents and passes them to the logical layer. The logical layer finds a combination of trajectories $\mathcal{S}_{\mathcal{T}}$ that fulfills all scenario constraints, like traffic rules, and furthermore fits best to the scenario description. To extend the parameter space covered by the simulation, this trajectory selection process can furthermore be randomized. The selected trajectories then are applied to kinematic vehicle models and smoothened using a Kalman filter. Thus, the choice of the MTP network is not restricted to models guaranteeing physical feasibility of the predictions. Based on the smoothened trajectories and the trajectory sent by the MPUT, which is applied to a kinematic vehicle model as well, the states of all road users are updated. The updated states are accessed by the evaluation module to assess the performance of the MPUT. Besides, new agents can enter the scenario through "spawn zones" or leave it through "target zones". Then, the simulation cycle is repeated until a termination condition of the scenario description is reached. 


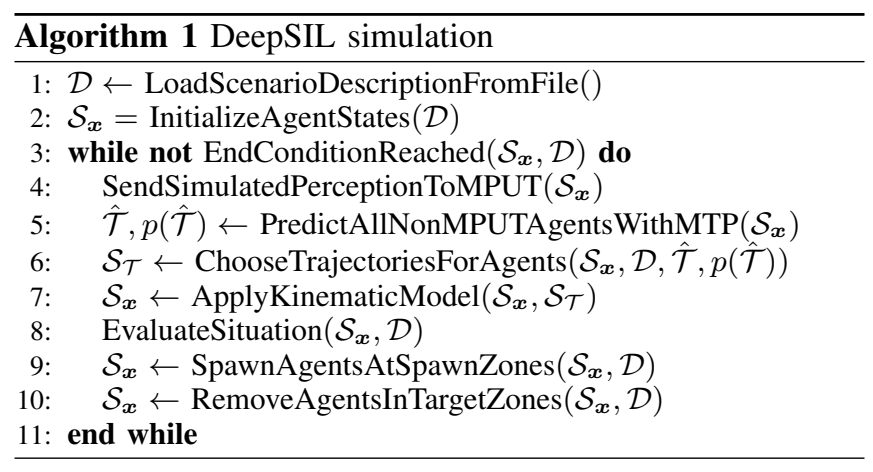

\section{A. Scenario Description}

The scenario description $\mathcal{D}=\left(\mathcal{M}, \mathcal{Z}_{\text {spawn }}, \mathcal{H}, \mathcal{C}, \mathcal{Z}_{\text {target }}\right)$ is basically comprised of two parts: first, a digital map $\mathcal{M}$, which features the lane geometry and the traffic rules, and secondly, a description of spawn zones $\mathcal{Z}_{\text {spawn }}$. By $\mathcal{Z}_{\text {spawn }}$, the number of road users involved in the scenario, their properties, and their initial states are controlled. Additionally, the scenario description contains further hyperparameters $\mathcal{H}$ that tailor the behavior of all road users in the scenario, a description of the evaluation metrics and criteria $\mathcal{C}$, and a description of the target zones $\mathcal{Z}_{\text {target }}$ defining conditions at which a road user can be removed from the simulation.

The map $\mathcal{M}$ is represented in the Lanelet2 [7] format, an open source map standard, which comprises the road geometry and the traffic rules, and allows to create routing graphs. In combination with the traffic rules, these routing graphs are used by the logic layer to identify conflicting lanes and to prioritize the road users.

The description of a spawn zone $\mathcal{Z}_{\text {spawn }, j} \in \mathcal{Z}_{\text {spawn }}$ can be formalized as

$$
\mathcal{Z}_{\text {spawn }, j}=\left(\boldsymbol{I}, \boldsymbol{f}(\theta), p_{\text {spawn }}(t), \boldsymbol{f}\left(\boldsymbol{x}_{0}\right), \mathcal{S}_{\mathrm{R}}\right),
$$

where $\boldsymbol{I}$ denotes the lanelet IDs, i.e. the road segments the spawn zone is located on, $\boldsymbol{f}(\boldsymbol{\theta})$ is the probability distribution over the static parameters like an agent's shape and class, $p_{\text {spawn }}(t)$ is the spawn probability at time $t, \boldsymbol{f}\left(\boldsymbol{x}_{0}\right)$ is the distribution over the initial states $x_{0}$, i.e. position, velocity, and acceleration, and $\mathcal{S}_{\mathcal{R}}$ is the set of routes.

A common special case is that the distributions are chosen as Dirac distributions. Thus, by defining a spawn zone for each agent with exactly specified $\boldsymbol{\theta}, \boldsymbol{x}_{0}$, and $t_{\text {spawn }}$, recorded scenarios can be reproduced concisely. Furthermore, $\boldsymbol{f}(\theta)$ and $\boldsymbol{f}\left(\boldsymbol{x}_{0}\right)$ are often specified as multinomial distributions, where certain parameter configurations are chosen with a defined probability. Alternatively, multidimensional Gaussian distributions or uniform distributions can also be used (except for the agent's class). $p_{\text {spawn }}(t)$ can be modeled as a binomial distribution in each time step. To avoid overcrowding the simulation, a maximum number of vehicles can be set as a constraint. Alternatively, $p_{\text {spawn }}(t)$ can be represented implicitly by a list of timestamps at which the road users are spawned. Thus, arbitrarily complex distributions can be modeled and recorded sequences can be reproduced.
A core part of $\mathcal{Z}_{\text {spawn, } j}$ is the set of routes

$$
\mathcal{S}_{\mathcal{R}}=\left\langle\mathcal{R}_{r}, p\left(\mathcal{R}_{r}\right)\right\rangle_{r=1, \ldots, M_{j}},
$$

which specifies a list of $M_{j}$ possible routes and their probabilities $p\left(\mathcal{R}_{r}\right)$. This list is only used for agents not controlled by an MPUT. Each route $\mathcal{R}_{r}$ corresponds to a sequence of abstract decisions like going left, right or straight at an intersection, or performing any other distinct action, such as performing a lane change on a highway, given a certain condition is met. To introduce some scenario variation, the simulator randomly chooses one of the $\mathcal{R}_{r}$, whenever a new

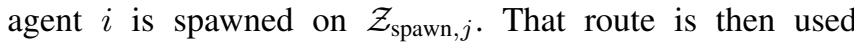
with $\mathcal{M}$ to generate a reference path $\mathcal{P}_{\text {ref }, i}$. In general, the reference path will consist of the centerlines of the lanelets that the selected route traverses, but can also be, e.g., a path for overtaking another vehicle. Note, however, that the realization of the trajectories along $\mathcal{P}_{\text {ref }, i}$ is still left up to the MTP network, which can account for the interactions between the agents, so that realistic trajectories are yielded.

\section{B. Multiple Trajectory Prediction Network}

DeepSIL can work with many neural networks for motion prediction, provided that they can predict one or more paths $\hat{\mathcal{T}}_{i, k}$ for a given agent $i$, where $k=1, \ldots, N_{i}$. The predicted paths should be a sequence of $\mathrm{x}$ - and $\mathrm{y}$-coordinates, and the network should predict the likelihood $p\left(\hat{\mathcal{T}}_{i, k}\right)$ for each path that the agent $i$ takes the respective path $k$. DeepSIL can provide the MTP network with the map, the current state of the agent, as well as its motion history, if required.

In our work, we use the network from our previous work [8]. It was trained on the Argoverse dataset [9], and produces state-of-the-art results. In contrast to [8], we do not feed state uncertainties to the neural network during training, since the data from the simulator comes with no uncertainty. Also, we use motion history subsets of random size during training, to ensure that the model can also predict accurately if it does not have a full two seconds of history available.

\section{Logic Layer and Randomization}

In our framework, we use the multiple predicted trajectories from the MTP network to our advantage by choosing a combination that complies with the scenario constraints and fits to the scenario description. The algorithm for selecting this combination is shown in Algorithm 2. We iteratively select trajectories and fix for the simulated road users from the set of predicted trajectories $\hat{\mathcal{T}}$, in a topological ordering $\mathcal{O}$ generated from a directed acyclic graph (DAG) $\mathcal{G}_{\text {DAG }}$. Fixing trajectories restricts the options for the remaining road users, since they should obey the scenario description, which also means to avoid collisions. Thus, we do not need to evaluate all possible combinations of trajectories. Rather, we can efficiently calculate a combination that complies with the scenario description.

To generate the DAG, we use the right of way traffic rules from the map to determine dependency relationships between the simulated road users. Each dependency represents an edge in the DAG. For example, a road user is dependent 
on a road user driving directly before it, and also on road users that have right of way over it at intersections with right of way. If the traffic rules do not yield a DAG directly, regularizing assumptions are applied to enforce that a DAG results. For example, at an unsignalized intersection, a firstcome-first-serve policy or simple randomization can be used to break up cycles.

To choose the trajectory for a given road user, we first transform the trajectory candidates $\hat{\mathcal{T}}_{i, k}$ for each agent $i$ into a Frenet frame referred to its reference path $\mathcal{P}_{\text {ref, } i}$ :

$$
\left(s_{i, k}, d_{i, k}\right)(t)=\mathcal{F}_{\mathcal{P}_{\text {ref }, i}}\left\{\hat{\mathcal{T}}_{i, k}\right\}
$$

Then, the resulting trajectories are filtered with a Kalman filter using the kinematic model (see section II-D). After this, we remove any trajectories that deviate too much from the reference path, i.e. $d_{\max , i}=\max _{t}\left\{\left|d_{i, k}(t)\right|\right\}>\delta$, where $\delta$ is a hyperparameter. If multiple trajectory candidates are left, a cost function is applied to select the trajectory

$$
J\left(\hat{\mathcal{T}}_{i, k}\right)=1-p\left(\hat{\mathcal{T}}_{i, k}\right)+\alpha_{d} d_{\max , i}+\alpha_{v}\left(v_{i, k}\left(t_{\text {eval }}\right)-v_{\text {tar }, i}\right)^{2} .
$$

Here, $\alpha_{d}$ and $\alpha_{v}$ are hyperparameters for the behavior of the simulated road users, and $v_{i, k}\left(t_{\text {eval }}\right)$ is the velocity at the evaluation time $t_{\text {eval }}=t_{\text {current }}+1 \mathrm{~s}$, where $v_{i, k}\left(t_{\text {eval }}\right)$ is obtained from the Kalman filter. The first term makes the logical layer prefer trajectories that are highly likely according to the MTP network, the second term punishes deviations $d_{\max , i}$ from the reference path, and the third term makes the logical layer prefer trajectories that adapt the road users's speed to the target velocity $v_{\operatorname{tar}, i}$. The target velocity can be a speed limit or may originate from a dynamic constraint. To cover a wider parameter space, the choice of hyperparameters can be randomized by either drawing $\delta, \alpha_{d}$, and $\alpha_{v}$ from a distribution or by directly selecting a possible trajectory randomly, e.g. according to $p\left(\hat{\mathcal{T}}_{i, k}\right)$.

We then check each candidate in order of ascending cost for collisions with trajectories of road users corresponding to parent vertices of the vertex of the current road user in the DAG. Using this scheme, in general, the simulated road users will move on trajectories predicted by the MTP network, but still respect the right of way according to the traffic rules. Only in the rare case that none of the predicted trajectories remain valid for a specific road user, the simulator has to resort to a fallback model, e.g. the IDM for one simulated road user following another one, or a simple $\mathrm{P}$ controller to stop at a yield line.

\section{Kinematic Model}

To model the motion of road users in $2 \mathrm{D}$, the kinematic bicycle model from [18] is used. However, in most practical situations, only the vehicles controlled by the MPUT are required to be modeled as such.

For vehicles that are simulated using the predictions from the MTP network, we use the Frenet transform (3) and move the vehicles in $1 \mathrm{D}$ along the reference path. For the 1D projection of the road users, the simple point-mass-model

$$
\dot{\boldsymbol{x}}(t)=\left[\begin{array}{lll}
0 & 1 & 0 \\
0 & 0 & 1 \\
0 & 0 & 0
\end{array}\right]\left[\begin{array}{l}
s \\
v \\
a
\end{array}\right]+\left[\begin{array}{l}
0 \\
0 \\
1
\end{array}\right] u
$$

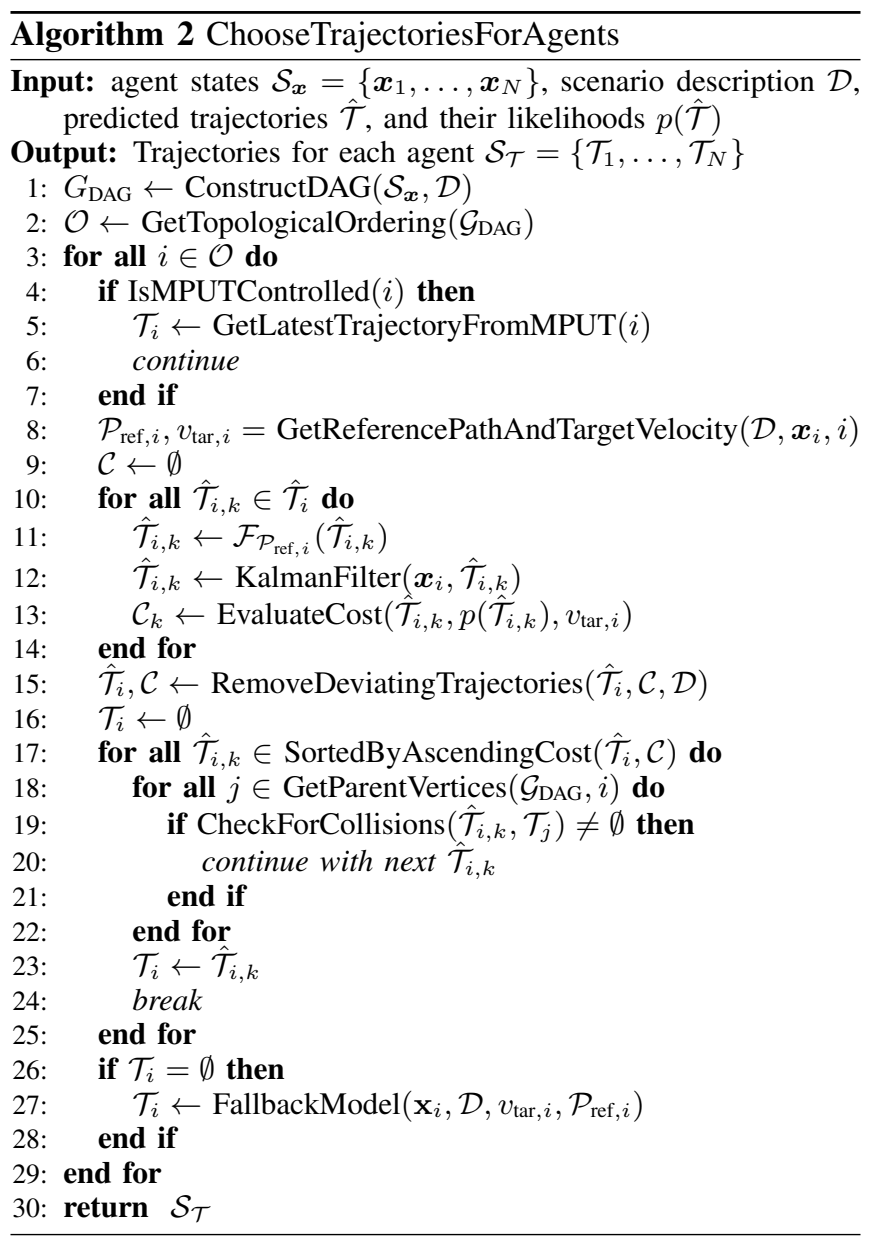

can be applied as the kinematic model, where $s, v, a$ and $u$ are the position, velocity, acceleration, and jerk along the reference path, respectively. This model requires less parameters than the kinematic bicycle model from [18], and, thus, is easier to parameterize in a scenario description. A Kalman filter is applied for the model (5) to smoothen the transition from the current to the next state, avoiding jumps in the velocity which do not comply with physical laws. This also makes the simulator more robust towards noisy predictions and, thus, increases the variety of MTP approaches that can be used with DeepSIL.

To model imperfect actuation, for the MPUT controlled vehicles, noise can be added to the control signals sent by the MPUT. Furthermore, additional controllers can be added to mimic the subsidiary control of the MPUT controlled vehicles. However, if such low-level control effects are not of interest for a particular simulation, like in our evaluation, instead, the trajectories sent by the MPUT can be interpolated and the respective control states can be applied directly. With that, computational effort can be saved.

\section{E. Simulation of Ego Perception and Ego State Estimation}

To emulate the perception of the vehicles controlled by the MPUT, the current states of all road users in the scene are accessed. However, to model limited visibility, e.g. due to 
limited sensor ranges, only a subset of these states is used to set up an environment model, which is sent to the MPUT for each controlled agent. Such an environment model contains an object list, where each object is described by its current state (position, velocity, and acceleration). To model measurement and detection uncertainty, random noise is added to the object states, clutter objects can be added to the object list or misdetected object can be omitted. By adding some Gaussian noise to the ego states of the MPUT-controlled vehicles, we simulate a localization module providing inputs to the MPUT. With these information, the MPUT can plan a trajectory for each assigned vehicle. Since the MPUT is allowed to control multiple vehicles, cooperative planning algorithms can also be tested with our simulation framework.

\section{F. Evaluation module}

To assess the behavior of the MPUT, an evaluation module can be used. This module can be configured to save the recorded trajectories of all simulated vehicles to a file for later detailed evaluation, but can also calculate configured metrics from the scenario description on the fly. Such metrics can be, e.g. the number of collisions that occurred, speed limit violations, high jerk values (indicating decreased passenger comfort), and/or time for maneuver completion.

\section{G. Implementation Details}

As a middleware between the different software components, we use the Robot Operating System (ROS) framework, which allows the modules to communicate via predefined messages and services. The software components can then be implemented in different programming languages. Our reference implementation of DeepSIL was done in the Python language. The MPUT, in our case, is implemented in $\mathrm{C}++$. We use the Lanelet2 API to load maps and traffic rules, and to construct reference paths from decision lists in the simulator. The neural network from [8] is implemented in PyTorch, and is executed by ONNX runtime with TensorRT acceleration. The synthetic images required for the MTP network are rendered directly on the GPU, where also the model inference takes place, which mainly consists of a convolutional neural network (CNN). This avoids copy operations between CPU and GPU. For common numbers of simulated vehicles, this allows for total prediction times less than 10 ms using an NVIDIA GeForce 1080 Ti GPU. For the scenario descriptions, we use YAML files, which specify the spawn zones, the path to the map file, and general parameters like the simulation step size.

\section{EVALUATION}

We evaluate our approach in two aspects. First, we present a case study, in which we show how this approach can be used to validate a motion planning scheme in a Monte-Carlo setting. Secondly, we validate that the simulator generates realistic trajectories by comparing it to real-world trajectories that were gathered at a complex T-junction using a traffic monitoring system. For both, our case study and the validation of DeepSIL, we use a scenario at a yield junction

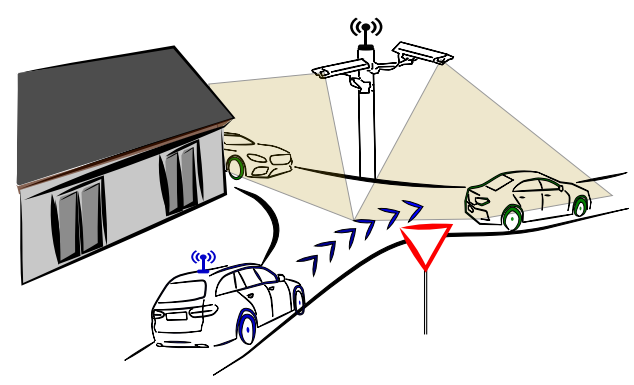

Fig. 2. Application Scenario (cf. [19])

in Ulm-Lehr in Germany. As sketched in Fig. 2, due to heavy occlusions from buildings, the yield junction presents a challenge for vehicles coming from the lane without right of way. In our case study, we evaluate a special motion planning scheme [19] from our previous work with respect to its decision making and its ability to dynamically merge into the junction using external perception. While in reality, the traffic surveillance system at the junction is used to resolve the occlusions [20], in this work, the data from the traffic surveillance system is used to validate that DeepSIL yields realistically simulated trajectories.

For the simulator hyperparameters, we use $\delta=5, \alpha_{d}=$ $0.05, \alpha_{v}=0.0167$. We lower $\alpha_{v}$ to 0.005 if a vehicle is approaching an intersection or junction and does not have right of way. The simulation step size is $50 \mathrm{~ms}$. We also simulate ideal perception and localization, i.e. no measurement noise is added.

\section{A. Case Study: Monte-Carlo Evaluation of a Motion Plan- ning Scheme}

To show that DeepSIL can be used effectively to test a motion planning scheme and yields plausible results, we use our motion planning scheme from [19] as MPUT. As a comparison, we use the results from [21], an earlier version of [19], that used an idealized simulation and planning scheme, which disregarded the road's curvature. For comparability, we reproduce the scenario from [21] as close as possible. The planning scheme [19] solves decision making and trajectory planning holistically, optimizing for risk and passenger comfort. For that, trajectory candidates are generated with an analytical solution, which then are checked to comply with the constraints, the cost of the valid candidates is calculated and the overall best candidate is selected.

For the chosen scenario, the goal of the MPUT is to dynamically merge a connected and automated vehicle into the yield junction, with two cars on the lane with right of way. To achieve this, the MPUT has to decide, whether to merge dynamically before the first car, into the gap between the two cars, or whether to stop at the yield junction and merge after the second car has passed. The behavior decision of the MPUT mainly depends on the (initial) gap size between the two vehicles. Hence, the probabilities $p_{\text {before }}, p_{\text {gap }}$, $p_{\text {gentle stop }}$ of the respective behavior decision are evaluated in dependence of the gap size. Furthermore, the probability 

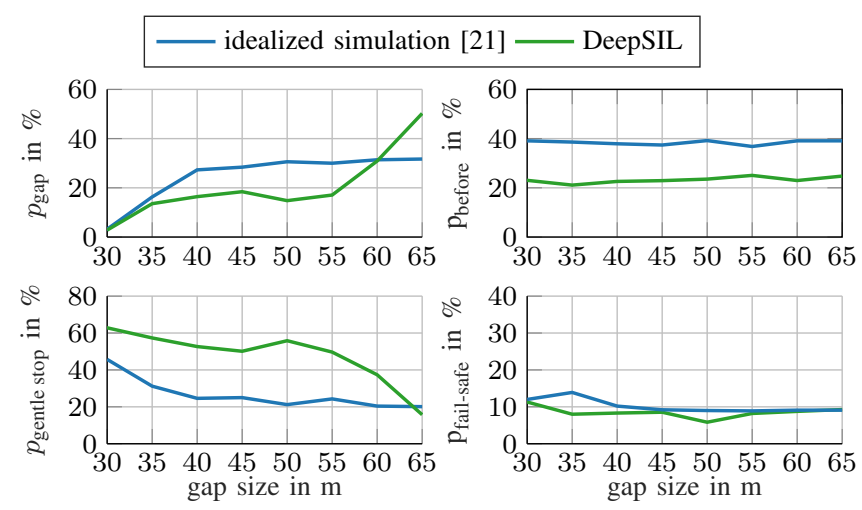

Fig. 3. Statistics on merging decision in dependence of the traffic gap between the two other vehicles.

$p_{\text {fail-safe }}$ is evaluated that the MPUT has to brake with about $3.3 \mathrm{~m} \mathrm{~s}^{-2}$. The initial gap size is controlled by the setting of the spawn zones of the other two vehicles, while the MPUT controlled vehicle is spawned at $65 \mathrm{~m}$ from the junction with an initial velocity sampled uniformly from the interval $v_{0, \text { MPUT }} \in[25,30] \mathrm{km} \mathrm{h}^{-1}$. Respectively, the initial states of the other two cars are drawn from normal distributions with mean $30 \mathrm{~km} \mathrm{~h}^{-1}$ and standard deviation $1 \mathrm{~km} \mathrm{~h}^{-1}$. The gap sizes are sampled uniformly between 25 and 65 metres. Thus, a broad variety of different timings is simulated.

Figure 3 shows the results after evaluating 5000 simulation runs with the time weight parameter of the MPUT set to $w_{t}=25$, like in [21]. A separate collision check by the evaluation module of the simulator did not show any collision. As compared to [21], the general behavior that bigger gap sizes increase the probability of merging, while stopping is less frequent, is reproduced. Furthermore, we observe a similar fail-safe probability of about $10 \%$. However, with DeepSIL, a far more realistic simulation is possible: first, the DeepSIL simulation accounts for the steep curvature of the road, so the MPUT has to slow down significantly to about $15 \mathrm{~km} \mathrm{~h}^{-1}$, which is considered in the newer planning scheme. Secondly, the other vehicles drive more realistically and slow down in the curve as well. Thus, at the junction the actual gap sizes become much smaller than they initially were. This narrows down the solution set for the MPUT and, thus, as compared to [21], the MPUT requires bigger gaps to merge and merging before the first vehicle is much less likely. In total, while the general behavior of [21] was still reproduced, DeepSIL provides a much more realistic environment for the MPUT, which leads to slightly different, but expected results.

Simulating the 5000 scenarios took about 9.5 hours, while the simulated time covered about 26 hours. Hence, we reach a speed up factor of about 2.7, where the MPUT appeared to be the speed bottleneck, with an average calculation time of $20 \mathrm{~ms}$ per planning cycle of $100 \mathrm{~ms}$.

\section{B. How Realistic is the DeepSIL Simulation?}

In order to evaluate how realistic the trajectories are that the simulator produces, we need to compare them to ground-

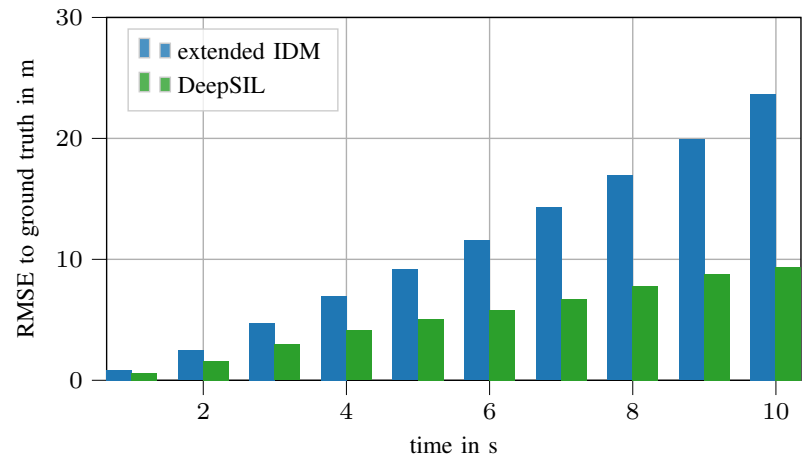

Fig. 4. Simulation errors over time, compared to the ground truth trajectory, averaged over the whole dataset of real-world traffic. Time is measured relative to the spawn/occurrence times of the respective vehicles.

truth data. We use recordings from the traffic surveillance system that span over a total of 128 minutes and contain a total of 643 tracks. The recordings contain data from different times of the day and varying traffic, with many complex interactions on the junction. The data comprises 3D box detections from two stereo camera setups, and four LiDAR systems. The detections were fused and tracked using a Kalman filter approach with forward-backward tracking to obtain accurate ground truth tracks. We then generated a scenario description, where for each track a simulated vehicle will be spawned that has the same initial states and makes the same abstract behavior decisions (e.g. left or right turn at an intersection or junction) as the respective ground truth track. The behavior decision is determined from the path taken by the respective track. After a vehicle has spawned, the simulator will simulate its trajectory for a configurable amount of time, or until it leaves the map. Only knowing the initial states and the abstract behavior decisions, the simulator generates the vehicles' trajectories, accounting for possible interactions with vehicles that are at an intersection at the same time, and saves the trajectories that the simulated vehicles took.

As a reference, we simulate the same scenario using a simple model which could also be used to test a motion planner: In the reference model, we use the IDM if the vehicle can drive freely or drives behind another vehicle, and the model from SUMO [2] at intersections and junctions, to correctly prioritize and schedule the traffic. We refer to that model as extended IDM.

To evaluate our approach against the extended IDM, we compare the recorded positions from the simulator to the ground truth positions from the traffic surveillance system. In Fig. 4, we show the root mean square (RMSE) position error over (simulated) time, averaging over all available data. As can be seen, our DeepSIL approach results in much lower error than the extended IDM. This is mainly due to the fact that the MTP network from [8] generates much more humanlike trajectories, since it was trained on a large dataset of real-world trajectories. As can be seen in Fig. 5, the model can produce trajectories that are very similar to the ground 


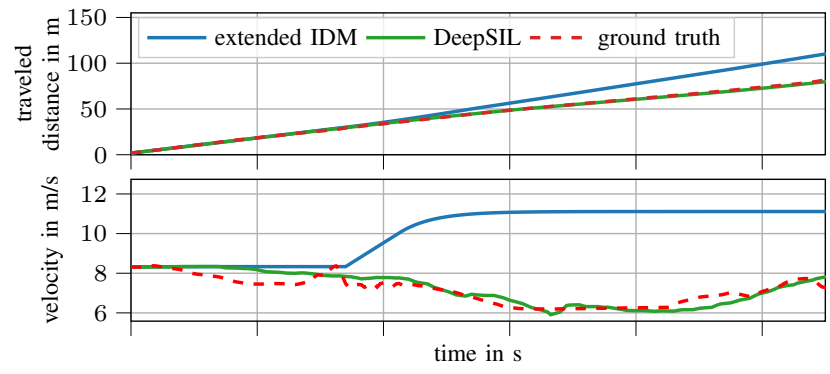

Fig. 5. Exemplary simulated trajectories from both DeepSIL and the extended IDM, compared to the real-world ground truth trajectory.

truth trajectories, for example when slowing down in a curve and accelerating after the curve. Overall, our model has a significantly higher accuracy compared to the extended IDM, while still correctly simulating interactions between the vehicles and avoiding collisions.

Besides the accuracy, the experiments highlight the good generalization properties of our approach. The used MTP network was trained on the Argoverse dataset, which contains neither class/shape information of the road users, nor the traffic rules or information about occlusions, e.g. due to buildings. In contrast, for our challenging scenario, occlusions play an important role and a broad variety of road user classes are observed in our recordings. Furthermore, the Argoverse dataset was recorded in the large US cities Miami and Pittsburgh, where the road users likely drive differently than on our German suburban T-junction. Yet, due to the flexibility and generic interfaces of DeepSIL, future developments of MTP networks can easily be integrated to improve the performance even more.

\section{CONCLUSIONS AND FUTURE WORK}

In this work, we proposed an architecture for a deep SIL simulator to test motion planning schemes. This allows to combine any suitable MTP network with the proposed logic layer, to create a very realistic microscopic simulation, that can be used to test motion planning software from scenario descriptions. We have demonstrated in a case study that it fulfills this purpose very effectively. Also, we have shown that the simulated trajectories are much more realistic than common simpler models by comparing them to ground truth recordings from a traffic surveillance system. Our approach is general enough to work with many multiple trajectory prediction networks, and, importantly, can work with imperfect predictions, but will greatly benefit from further advancements in the field of motion prediction. In our future work, we want to expand the scheme to work with motion prediction networks that can use traffic light state data, so that signalized intersections can be simulated more accurately.

\section{REFERENCES}

[1] S. Riedmaier, T. Ponn, D. Ludwig, B. Schick, and F. Diermeyer, "Survey on scenario-based safety assessment of automated vehicles," IEEE Access, vol. 8, pp. 87 456-87477, 2020.
[2] P. A. Lopez, M. Behrisch, L. Bieker-Walz, J. Erdmann, Y.-P. Flötteröd, R. Hilbrich, L. Lücken, J. Rummel, P. Wagner, and E. Wießner, "Microscopic traffic simulation using sumo," in Proc. IEEE Int. Conf. Intell. Transp. Syst. (ITSC). IEEE, 2018.

[3] M. Treiber, A. Hennecke, and D. Helbing, "Congested traffic states in empirical observations and microscopic simulations," Physical review $E$, vol. 62 , no. 2 , p. $1805,2000$.

[4] J. Ho and S. Ermon, "Generative adversarial imitation learning," in Proc. Int. Conf. Neural Information Processing Syst. (NIPS), 2016, pp. 4572-4580.

[5] R. P. Bhattacharyya, D. J. Phillips, B. Wulfe, J. Morton, A. Kuefler, and M. J. Kochenderfer, "Multi-agent imitation learning for driving simulation," in Proc. IEEE/RSJ Int.Conf. Intell. Robots Syst. (IROS), 2018, pp. $1534-1539$.

[6] R. P. Bhattacharyya, D. J. Phillips, C. Liu, J. K. Gupta, K. DriggsCampbell, and M. J. Kochenderfer, "Simulating emergent properties of human driving behavior using multi-agent reward augmented imitation learning," in Proc. Int. Conf. on Robotics and Automation (ICRA), 2019, pp. 789-795.

[7] F. Poggenhans, J.-H. Pauls, J. Janosovits, S. Orf, M. Naumann, F. Kuhnt, and M. Mayr, "Lanelet2: A high-definition map framework for the future of automated driving," in Proc. IEEE Int. Conf. Intell. Transp. Syst. (ITSC), Hawaii, USA, 2018.

[8] J. Strohbeck, V. Belagiannis, J. Müller, M. Schreiber, M. Herrmann, and M. Buchholz, "Multiple trajectory prediction with deep temporal and spatial convolutional neural networks," in Proc. IEEE/RSJ Int.Conf. Intell. Robots Syst. (IROS), Virtual, 2020.

[9] M.-F. Chang, et al., "Argoverse: 3d tracking and forecasting with rich maps," in Proc. IEEE/CVF Conf. Computer Vision and Pattern Recognition (CVPR), 2019, pp. 8748-8757.

[10] M. Klischat, E. I. Liu, F. Holtke, and M. Althoff, "Scenario factory: Creating safety-critical traffic scenarios for automated vehicles," in Proc. IEEE Int. Conf. Intell. Transp. Syst. (ITSC), 2020, pp. 1-7.

[11] C. Pek, M. Koschi, and M. Althoff, "An online verification framework for motion planning of self-driving vehicles with safety guarantees," in AAET-Automatisiertes und vernetztes Fahren, 2019.

[12] S. Kitajima, K. Shimono, J. Tajima, J. Antona-Makoshi, and N. Uchida, "Multi-agent traffic simulations to estimate the impact of automated technologies on safety," Traffic injury prevention, vol. 20, no. sup1, pp. S58-S64, 2019.

[13] A. Dosovitskiy, G. Ros, F. Codevilla, A. Lopez, and V. Koltun, "CARLA: An open urban driving simulator," in Pro. Ann. Conf. on Robot Learning, 2017, pp. 1-16.

[14] M. Naumann, F. Poggenhans, M. Lauer, and C. Stiller, "CoInCarSim: An open-source simulation framework for cooperatively interacting automobiles," in Proc. IEEE Int. Conf. Intelligent Vehicles(IV), Changshu, China, 2018, pp. 1879-1884.

[15] A. Pütz, A. Zlocki, J. Küfen, J. Bock, and L. Eckstein, "Database approach for the sign-off process of highly automated vehicles," in Int. Tech. Conf. Enhanced Safety Vehicles (ESV), 2017.

[16] H. Cui, V. Radosavljevic, F. Chou, T. Lin, T. Nguyen, T. Huang, J. Schneider, and N. Djuric, "Multimodal trajectory predictions for autonomous driving using deep convolutional networks," in Proc. Int. Conf. on Robotics and Automation (ICRA), 2019, pp. 2090-2096.

[17] J. Colyar and J. Halkias, "US highway 101 dataset," Federal Highway Administration (FHWA), Tech. Rep. FHWA-HRT-07-030, pp. 27-69, 2007.

[18] F. Gritschneder, K. Graichen, and K. Dietmayer, "Fast trajectory planning for automated vehicles using gradient-based nonlinear model predictive control," in Proc. IEEE/RSJ Int.Conf. Intell. Robots Syst. (IROS), Madrid, Spain, 2018, pp. 7369-7374.

[19] J. Müller, M. Herrmann, J. Strohbeck, and M. Buchholz, "Motion planning for connected automated vehicles at occluded intersections with infrastructure sensors," submitted to IEEE Trans. Intell. Transp. Syst., 2020.

[20] M. Buchholz, J. Müller, M. Herrmann, J. Strohbeck, B. Völz, M. Maier, and R.-W. Henn, "Handling Occlusions in Automated Driving by Infrastructure Sensors: A V2X Approach with a MEC Server-based Environment Model," IEEE Intelligent Transportation Systems Magazine, 2021, accepted for publication.

[21] J. Müller and M. Buchholz, "A risk and comfort optimizing motion planning scheme for merging scenarios," in Proc. IEEE Int. Conf. Intell. Transp. Syst. (ITSC), Auckland, New Zealand, 2019, pp. 31553161. 